\title{
POLYMER-BASED ELECTROSPRAY CHIPS FOR MASS SPECTROMETRY
}

\author{
Xuan-Qi Wang, Amish Desai, Yu-Chong Tai, Lawrence Licklider", Terry D. Lee* \\ California Institute of Technology, Pasadena, CA 91125, USA \\ *Beckman Research Institute, City of Hope Research Center, Duarte, CA91010, USA
}

\begin{abstract}
In this paper, we present our development of a MEMS chip with an overhanging polymer microcapillary $2.5 \mathrm{~mm}$ in length and with a $5 \mu \mathrm{m} \times 10 \mu \mathrm{m}$ orifice size at the tip. The fabricated chips have been successfully interfaced with a mass spectrometer (MS) to validate electrospray ionization (ESI) for biochemical analysis. The prediction of a reduction in Taylor cone size has also been observed with real time ESI fluid visualization from our chip. Built-in micro particle filters and centimeter long serpentine micro channels were fabricated on the chip with a low temperature process by using the Parylene polymer as a structural material, aluminum and photoresist as sacrificial layers, and bromine triflouride $\left(\mathrm{BrF}_{3}\right)$ gas phase etching for final microcapillary releasing. The use of an overhanging polymer structure adds a new a level of mechanical robustness that was never achievable with other thin films. Functionality of our device was proven by consistent detection of Myoglobin in a $200 \mathrm{nM}$ solution at a flow rate of $35 \mathrm{~nL} / \mathrm{min}$ and a voltage potential of $1.5 \mathrm{kV}$. This MS interface chip represents vital and significant improvements in MEMS process technology and MS functionality with respect to the silicon nitride $\left(\mathrm{Si}_{\mathrm{x}} \mathrm{N}_{y}\right) \mathrm{ESI}$ nozzles previously reported by our group [1].
\end{abstract}

\section{INTRODUCTION}

Over the past decade there has been increasing interest in the development of miniaturized chemical analysis systems using MEMS technology. However, due to the diversified process of chemical/biochemical analysis and a wide range of samples involved, a self-contained mass produced "lab on a chip" with the same economy of scale as electronic components has yet to be realized. Although such a "lab on a chip" is not a commercial product today, the vital components such as MEMS chromatographs, and MEMS capillary electrophoresis required for such a system have been under extensive development. Consequently, an important task then, is to use these existing MEMS components and insert them with commercially available macroscopic instrumentation.
Mass spectrometers are one type of widely used macroscopic instrument for the analysis of biomolecuar structures such as proteins, carbohydrates, lipids and polynucleotides. With its ability to analyze large proteins and peptides combined with its femtomole sensitivity and versatility (minimal sample preparation) the MS is widely used. Even through current MS are still large compared to MEMS systems, the small sample volume requirement and high detection sensitivity is ideally suited for coupling with MEMS devices.

One simple method by which MEMS chemical systems can generate ions for MS analysis is with electrospray ionization (ESI) [2][3]. ESI is efficient, gentle, and easily coupled to liquid chromatography and capillary electrophoresis separations. It is an atmospheric pressure ionization technique in which ions of peptides and proteins below 70,000 $\mathrm{Da}$ can be generated. Compared to other ionization methods [5], ESI advantages include: quick and softest ionization, ease of use, and complete compatibility with liquid chromatography. It is an atmospheric pressure ionization technique in which gaseous ionized molecules are produced in a fine spray from a liquid droplet in the presence of a strong electric field between the capillary tip and MS inlet. The released ionized molecules are then directed into the MS inlet by the applied field. In short, the applied electric field changes the shape of the fluid droplet from a spherical shape to a cone called the Taylor cone. A theoretical description of the electrostatic dispersion in "Taylor cone" formed by electrospray can be found in [4]. The base of this cone is dependent on the geometry and the wetted area (wall thickness) of the capillary. Hence, reduction of these parameters reduces the size of the Taylor cone, thus reducing dead-volume. The most distinct advantage of ESI over other ionization methods is that it can form a simple and direct way to interface MEMS device and mass spectrometers. All that is required on the MEMS chip is an electrospray nozzle to the mass spectrometer inlet.

As a first step in investigating the feasibility of a MEMS interface between the chip and the MS, our group previously developed a micron-sized electrospray nozzle and demonstrated its application for ESI MS. Although the first effort eliminated the problems of the traditional complex preparation of a fused silica capillary, the silicon 
nitride overhanging technology had the following disadvantages: a) it was a complex process, b) it had yield problems due to $\mathrm{Si}_{\mathrm{x}} \mathrm{N}_{\mathrm{y}}$ intrinsic stress, c) it required high temperature thin films and last, d) it had an overhanging length limitation due to the sacrificial etching material. The purpose of this effort is to overcome limitations of our silicon nitride ESI tips and demonstrate the utility and robustness of overhanging polymer microcapillary technology for a MS-ESI interface. In addition to discussing the overhanging polymer process issues, we also validate with real-time fluid visualization of the electrospray functionality of our nozzles. The expected improvements in the functionality of the electrospray nozzles are demonstrated by real-time visualization of the fluid Taylor cone and by MS analysis of a protein sample.

\section{OVERHANGING POLYMER MICROCAPILLARY TECHNOLOGY}

The overhanging capillary technology has several advantages for ESI over the blunt-edged orifice [6] [7] (Fig. 1). An overhanging capillary (Fig.1C) that extends away from the bulk of the chip minimizes interference with the electric field required for electrospray. From Fig. $1 \mathrm{C}$, we can see that the length and side-profile of our current polymer microcapillary greatly improves upon the $\mathrm{Si}_{\mathrm{x}} \mathrm{N}_{\mathrm{y}}$ structures. The polymer microcapillary length is $2.5 \mathrm{~mm}$ vs. $40 \mu \mathrm{m}$ for the nitride tips. In fact, the advantages of overhanging ESI structures have been affirmed by Foret et al. [8] who integrated the blunt glass channel orifice arrays with conventionally pulled fused silica capillary (FSC) tips. Ignoring the fact that the integration with the FSC capillaries was performed by a tedious gluing process, these FSC capillaries increase dead volume, increase wetted area at the tip, and have low ES efficiency. Shortcomings of conventional ESI (FSC tips) can be found in our previous work[1]. In comparison, the use of a microcapillary type structure results in minimal wetted surface area at the tip, which becomes important for reducing the volume of the Taylor cone (see Discussion section).

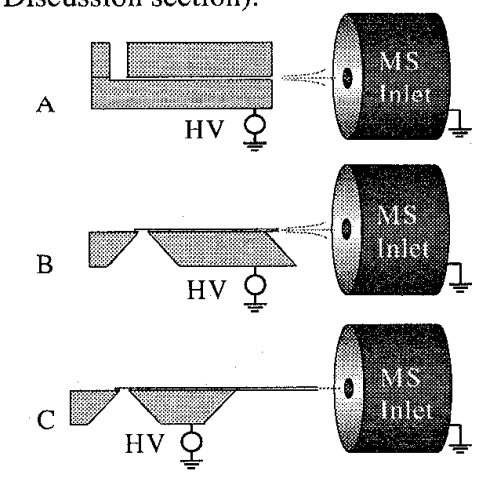

Fig.1: Comparison of ESI chip side-wall profiles and tip distance to MS inlet. A) glass channel structure B) our previous $S i_{x} N_{y}$ tips C) new polymer microcapillaries.

Our earlier $\mathrm{Si}_{\mathrm{x}} \mathrm{N}_{\mathrm{y}}$ ESI tips suffered from numerous disadvantages. For example, the outward sloping sidewall combined with the short length of the silicon nitride nozzle limited the tip to inlet distance as well as interfering with the electric field profile at the tip (Fig.1B). Furthermore, it is well known that fabrication of mm-long silicon nitride overhanging structures pose significant challenges. $\mathrm{Si}_{\mathrm{x}} \mathrm{N}_{\mathrm{y}}$ capillaries longer than a few hundred microns curved significantly as shown in Fig.2 due to film stress gradients (typically 200MPa for LPCVD $\mathrm{Si}_{\mathrm{x}} \mathrm{N}_{\mathrm{y}}$ ). To achieve channels longer than a few hundred microns long, etching holes on $\mathrm{Si}_{\mathrm{x}} \mathrm{N}_{\mathrm{y}}$ channels were needed and had to be sealed after sacrificial layer etching. These etch holes not only complicated the process, but the resulting stress concentration around the etching holes caused the released microchannels to crack as shown in Fig. 3. Finally, the fragile silicon nitride capillaries could shatter with the slightest contact with MS inlet during handling.

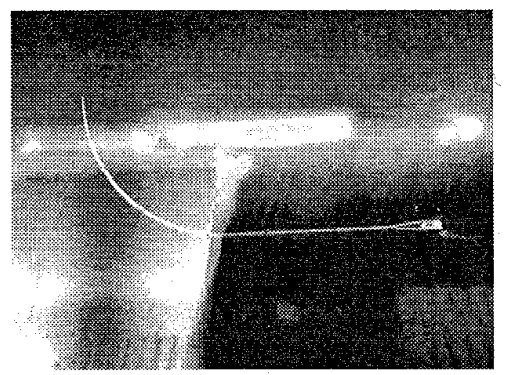

Fig.2: Stress on $1.5 \mathrm{~mm}$ long silicon nitride nozzle causes extreme curvature.

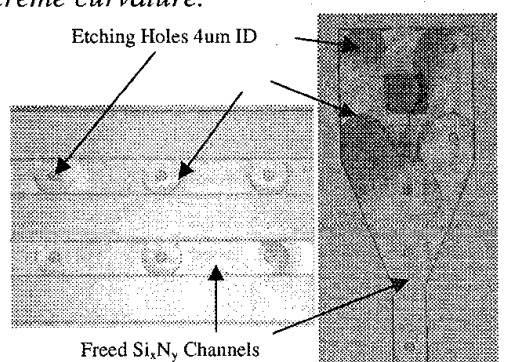

Fig.3: Cracking due to stress in $S i_{x} N_{y}$ micro-channels after sacrificial layer etch (same chip as Fig. 2).

Many of the drawbacks of our $1^{\text {st }}$ generation $\mathrm{Si}_{\mathrm{x}} \mathrm{N}_{\mathrm{y}}$ ESI tips are eliminated with our new overhanging polymer technology. In order to demonstrate the 
feasibility of a overhanging polymer structure here, we have utilized Parylene-C. As a bio-compatible polymer material, Parylene-C has been used to make MEMS microchannels [9] and microvalves[10]. In contrast to the $\mathrm{Si}_{\mathrm{x}} \mathrm{N}_{\mathrm{y}}$ process mentioned before, polymer material such as Parylene is ideal for fabrication of high aspect ratio overhanging structures due to its low tensile stress $(<100 \mathrm{Mpa})[11]$. Since the deposition is at room temperature, photoresist can be used as the sacrificial material. Unlike PSG etching, this resist sacrificial etching (with acetone) does not require any etch holes along the channel, and thus, structures that are orders of magnitude longer can be easily fabricated. The Young's modulus of Parylene $(\sim 3 \mathrm{GPa})$ is only about one hundredth of $\mathrm{Si}_{\mathrm{x}} \mathrm{N}_{\mathrm{y}}$. In terms of robustness, the fabricated Parylene microcapillaries are far superior. $\mathrm{Si}_{\mathrm{x}} \mathrm{N}_{\mathrm{y}}$ capillaries can shatter easily with the slightest contact with other objects, but the Parylene capillaries although 600 times longer, flex and return to their original shape. Fig. 4 illustrates the Parylene ESI tips' amazing plasticity. Fig. 5 shows the same chip with unbroken capillaries after more than 20 such tests. In spite of the long overhanging structure, quite a few chips can even survive table-top falls and mishandling without having the tips break. To sum up, the robustness of the parylene nozzles, and the simple process makes this technology most attractive.

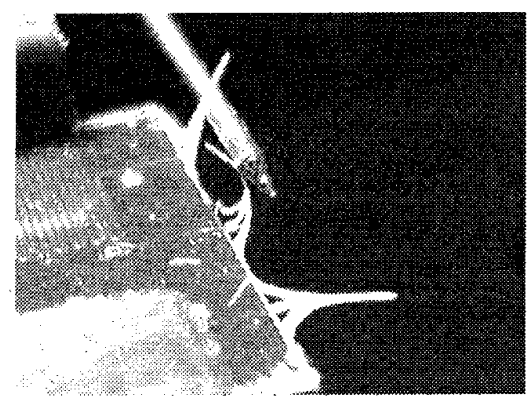

Fig. 4: Mechanical robustness demonstrated by deflection with a probe tip.

We must point out, however, that there are other micromachining techniques of constructing microcapillaries, but they suffer similar problems in comparison with this low temperature polymer process. Talbot and Pisano [12] demonstrated a two-wafer polymolding process to make polysilicon microcapillary. It is not easy to integrate these needles with microchannels and other microfluidic components on chip. Papaustky et al. [13] reported a low temperature process to fabricate surface micromachined overhanging metallic microchannels. The metallic structure material makes it difficult to perform some of the on-chip separation methods (such as electrophoresis), and to visualize the liquid flow in the channels. In our literature search, no polymer-based electrospray overhanging microcapillary has been reported before.

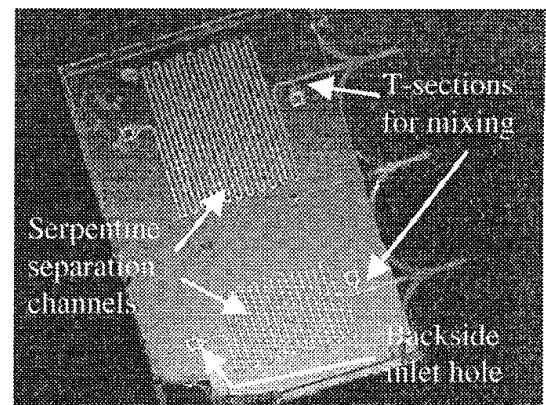

Fig.5: A microchip with $5 \mathrm{~cm}$ and $10 \mathrm{~cm}$ long serpentine channels with T-sections, inlets and outlets integrated with $2.5 \mathrm{~mm}$ long overhanging ESI micro-capillaries.

\section{DESIGN AND FABRICATION}

The silicon micromachined channels and capillaries consists of a "sandwich" of a $5 \mu \mathrm{m}$ thick photoresist enclosed by two Parylene layers each $3 \mu \mathrm{m}$ thick on a 500 $\mu \mathrm{m}$ thick silicon substrate. The overhanging structure is created by gas phase etching of the $\mathrm{Si}$ membrane underneath. Several variations were designed. Figs. 5 and 6 show a $1 \mathrm{~cm} \mathrm{x}, 0.7 \mathrm{~cm}$ die with 5 and $10 \mathrm{~cm}$ long channels for separation, backside inlets, and T-sections for mixing or adding other liquids. Figs. 7 and 8 show the orifice dimensions and the feature of the parylene polymer surface. Note that the surface roughness on the underside of the tip is due to the roughening of the Si substrate with $\mathrm{BrF}_{3}$ (Fig. 8).

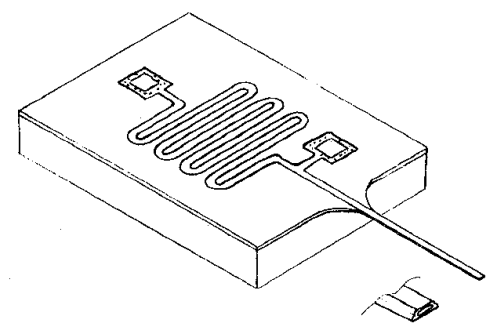

Fig. 6: Schematic of structure with serpentine channel, two backside inlets, and an overhanging microcapillary ESI interface.

The major fabrication process is shown in Fig. 9. First, we open windows in silicon wafers coated with 1.5 $\mu \mathrm{m}$ silicon dioxide on both sides. Next, the backside cavities are etched by $\mathrm{KOH}$ to leave a $10 \mu \mathrm{m}$ silicon membrane left using $\mathrm{SiO}_{2}$ as a mask layer. The $\mathrm{SiO}_{2}$ layer on wafer top side is patterned and etched by $\mathrm{BHF}$. $\mathrm{BrF}_{3}$ 
gas phase etching follows to roughen the silicon surface for adhesion enhancement. Next, a $3 \mu \mathrm{m}$ thick Parylene-C is deposited on the wafer front side only. After patterning the Parylene with oxygen plasma, a $5000 \AA$ thick $\mathrm{Al}$ layer is evaporated and patterned to form the filter region.

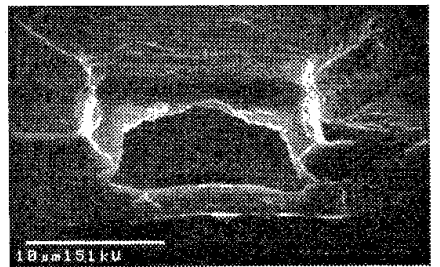

Fig.7: Front view of a microcapillary

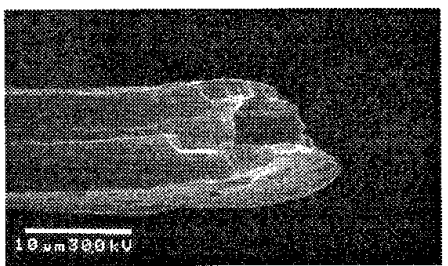

Fig. 8: Side view of a microcapillary

Photoresist layer of $5 \mu \mathrm{m}$ thick is spun and patterned. The wafer is then hard baked at $120^{\circ} \mathrm{C}$ for 10 minutes to evaporate the solvent in the photoresist. This step is important, otherwise the channel will tend to buckle and burst if heat is applied later on. A $3 \mu \mathrm{m}$ thick Parylene-C layer is then deposited on the front side only followed by $0.1 \mu \mathrm{m} \mathrm{Al}$ evaporated and patterned as a masking layer. Afterwards, oxygen plasma etching defines the microcapillary tip. Next, the wafer is diced, and the thin silicon membrane is etched away by $\mathrm{BrF}_{3}$ to open up the backside inlet holes and release the overhanging polymer structure. Finally, the photoresist layer is dissolved away with acetone to open the channels. The chips are then rinsed and immersed in alcohol and deionized water for several hours.

To minimize tip clogging, particle filters are also incorporated in several other designs by employing a double sacrificial material method. Filter structures can be formed by using sub-micron channel heights in the filter areas, and larger channel heights for the remaining microchannel length. To achieve this type of bi-level sacrificial structure, careful control of both thickness' is essential. Therefore, in our process we use a sub-micron Al layer for the filter region and thicker photoresist for the rest of the structure. The flexibility of polymer must also be taken into account when designing structures that are subject to mechanical pressure. For example, fluidic pressure can cause deformation in the sub-micron filter area and nullify its effectiveness as a sub-micron filter. To address this deformation problem, polymer anchor structures are fabricated in this region to hold the top and bottom of the channel together.
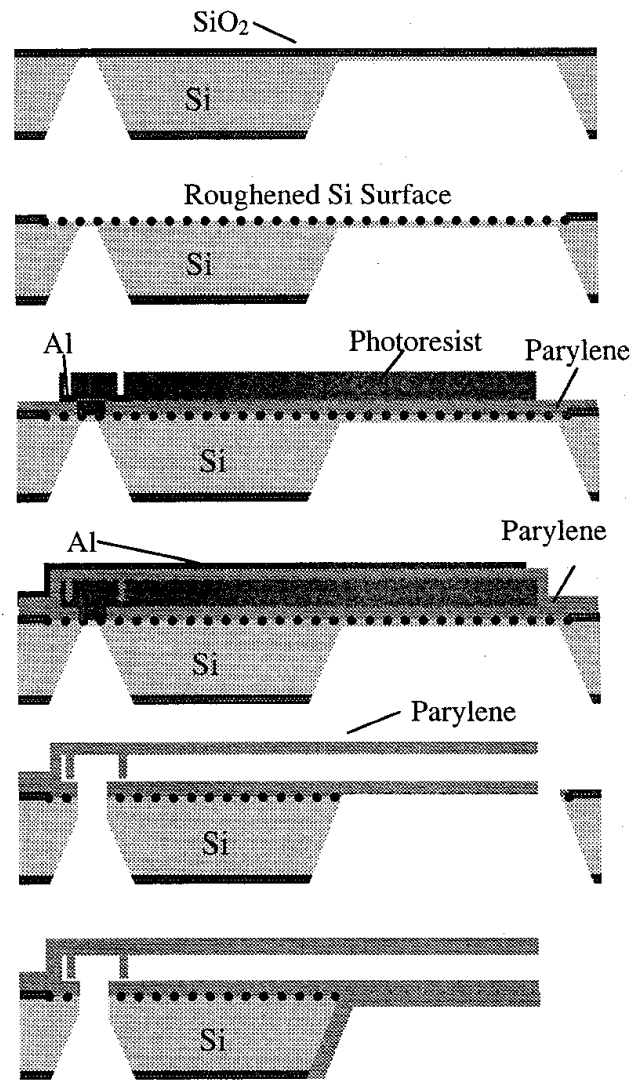

Fig 9: Process flow

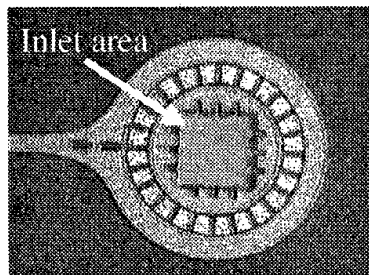

Before sacrificial etching

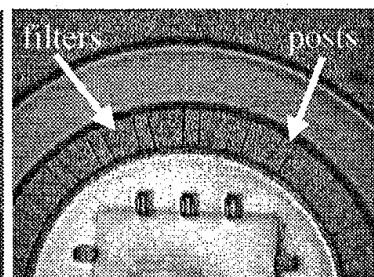

After sacrificial etching
Fig. 10: Inlet of Micro channel with integrated filters

Gas phase silicon etchant $\mathrm{BrF}_{3}$ is used to release the microcapillaries. This etching method [14] serves two purposes in this process: 1) to partially roughen the silicon surface for increased adhesion to the Parylene, and 2) to release the microcapillaries from silicon substrate in the last fabrication step. Compared to other silicon etching methods, plasma etching offers much less silicon undercutting and tends to damage the Parylene film on the chip. Even though KOH or TMAH do not attack Parylene 
at low temperature, they do attack the Parylene-silicon interface significantly and cause Parylene to peel off from the substrate. In contrast, the gas phase silicon isotropic etchant, $\mathrm{BrF}_{3}$, can etch silicon spontaneously at room temperature and maintain the integrity of Parylene-silicon interface.

\section{EXPERIMENTS}

With a high magnification lens, the Taylor cone formation at the orifice of the polymer micro-capillaries was video taped. The test solution consisted of $1 \%$ acetic acid (by volume) added to 1:1 $\mathrm{MeOH}$ :Water. The sequence of snapshots below clearly indicates how size of the Taylor cone is governed by the tip orifice geometry and the formation of the cone is governed by electrical potential. In fact, the cone size and shape is also related to flow rate and the distance between the tip and MS inlet. It is also important to note, that the view of the ESI is taken from a top view through a microscope, and that the "cone" most likely is not circular but flattened in the vertical dimension because the tip orifice is rectangular. From Fig. 11a and $11 \mathrm{~b}$ we note that although the electric field is applied the meniscus forces are strong enough to create the curvature. Subsequently, as the potential increases to $1250 \mathrm{~V}$, we see the generation of a sharp and stable cone(Fig. 11d). The distance from the tip to the inlet was roughly $500 \mu \mathrm{m}$. No plume is visible from the point of the cone, because droplet size is likely to be below $100 \mathrm{~nm}[4]$.

For final verification, a standardized $200 \mathrm{nM}$ Myoglobin solution was continually infused with a reservoir pressure of $2 \mathrm{psi}$ and $1.6 \mathrm{kV}$ potential. Fig. 12 shows the similarity of a mass spectrum analysis of myoglobin using the polymer microcapillary vs. conventional silica capillary. Both MS scans were performed using 15 attomoles of sample.

\section{DISCUSSION}

The ability to fabricate precise micrometer-sized tip geometries introduces further opportunities to examine micro-electrospray phenomena. Although a lot of experimental data and understanding has been acquired by various groups such as Fenn (1985) and Wilm (1994) regarding electrospray, mathematical models exist for only special cases.

$$
r_{e}=\left(\frac{\rho}{4 \pi^{2} \gamma\left[\left(\frac{U_{a}}{U_{t}}\right)^{2}-1\right] \tan \left(\frac{\pi}{2}-v\right)}\right)^{1 / 3} \cdot(d V / d t)^{2 / 3}
$$

For instance, the previous mathematical model developed by Wilm and Mann [6] models a circular cone with a specific cone tip angle, where $r_{e}$ represents the radius of the emission region at the tip of the Taylor cone, $\gamma$ the surface tension of the liquid, $\rho$ the density of the liquid, $\mathrm{U}_{\mathrm{a}}$ the applied voltage, $\mathrm{U}_{\mathrm{t}}$ the voltage at which the cone is formed, $v$ the cone angle, and $\mathrm{dV} / \mathrm{dt}$ the flow rate. The equation below predicts that $r_{e}$, the emission radius can be reduced with a reduction in flow rate. For our

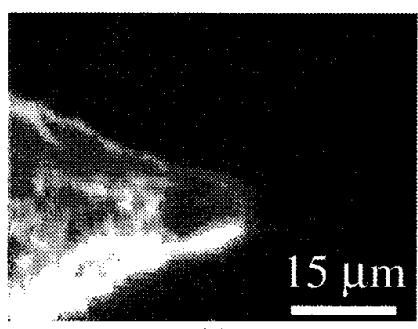

(a)

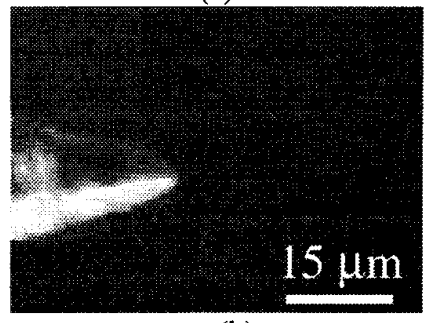

(b)

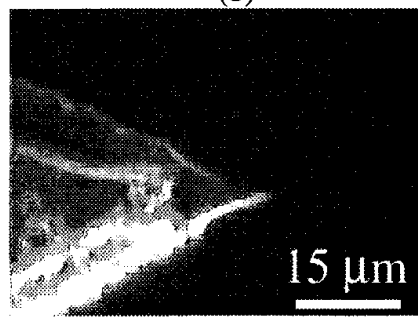

(c)

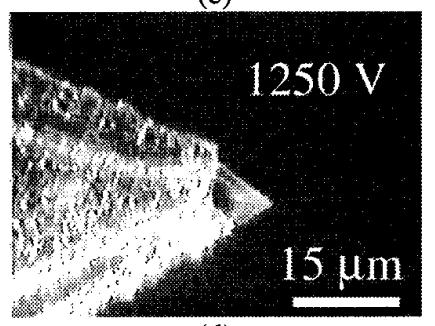

(d)

Fig. 11: Video snapshots of formation of Taylor cone on microcapillary as voltage is increased from $800 \mathrm{~V}$ to $1250 \mathrm{~V}$ (top to bottom).

microcapillary, the orifice is rectangular and initial measurements of the cone angle show significant deviation from that of a circular capillary Taylor cone 
$\left(\sim 49^{\circ}\right)$. Experiments are in progress to determine the extent to which ESI-MS performance is affected by the shape of the ES nozzle.

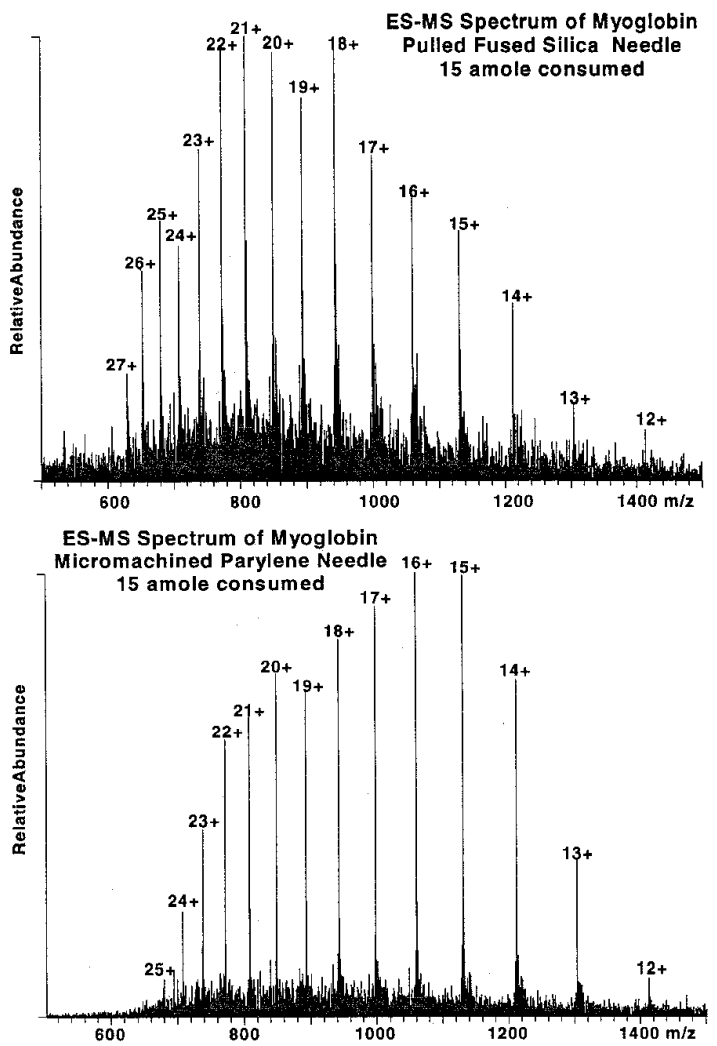

Fig. 12: Parylene microcapillary ESI performance (bottom) similar to conventional MS spectrum of Myoglobin.

\section{CONCLUSION}

This MS ESI interface chip represents vital and significant improvements in MEMS process technology and MS functionality with respect to the silicon-nitride ESI nozzles reported before. We validate here, the tremendous advantages and viability of Parylene polymer technology combined with gas phase etching for mm-long rugged freestanding structures. In addition, fluid visualization of the Taylor cone formation from our tips unearths new issues in the study of electrospray phenomena. As an example of MEMS technology for chemical analysis systems, the work presented here has made the MEMS electrospray chip more practical.

\section{ACKNOWLEDGMENTS}

This project is funded by NIH grant 2 R01 RR0621706 and by Cancer Center core grant P30 CA33752. The authors would also like to thank Mr. Trevor Roper for maintaining the equipment.

\section{REFERENCES}

[1] A. Desai, Y.C. Tai, M.T. Davis, T.D. Terry, "A MEMS Electrospray Nozzle for Mass Spectroscopy", Transducers ' 97.

[2] C.M. Whitehouse, R.N.Dreyer, M.Yamashita, J.B.Fenn, "Electrospray interface for LC and MS," Anal. Chem. 1985.

[3] Ymamshita, J.B.Fenn, "Electrospray Ion Source. Another Variation on the Free-Jet Theme,"J.Phys.Chem. 1984.

[4] M.Wilm, M.Mann, "Electrospray and taylor cone theory, Dole's beam of macromolecules at last?" Intl. J. of Mass Spectr.\& Ion Proc. 1994, 167-180.

[5] B. Siuzdak, "Mass Spectrometry for Biotechnology." Academic Press, 1996.

[6] Q. Xue, F. Foret, Y. Dunayevskiy, P. Zavracky, N. McGruer, B. Karger, "Multichannel Microchip Electrospray Mass Spectrometry," Anal. Chem. 1997, $69,426-430$

[7] R. Ramsey, J Ramsey, "Generating Electrospray from Microchip Devices Using Electroosmotic Pumping," Anal. Chem. 1997, 69, 1174-1178

[8] F.Foret, H.Liu, B.Zhang, C.Felten, B.L.Karger, "Microdevices for electrospray mass spectrometry." Micro Total Analysis Systems'98.

[9] P.F.Man, D.K. Jones, and C.H.Mastrangelo, "Microfluidic plastic capillaries on silicon substrates: A new inexpensive technology for bioanalysis chips." MEMS'97.

[10] X.Yang, C.Grosjean, and Y.C. Tai, "A low power MEMS silicone/parylene valve", Solid-State Sensor and Actuator Workshop, 1998.

[11] S.Dabral, J. Van Etten, X.Zhang, C.Apblett, G.R. Yang, P.Ficalora and J.F.Mcdonald, "Stress in thermally annealed parylene films." Journal of Electronic Materials, Vol.21, No.10 1992.

[12] N.H. Talbot and A.P. Pisano, "Polymolding: Two wafer polysilicon micromolding of closed-Flow Passages for microcapillaries and microfluidic devices". Solid-State Sensor and Actuator Workshop, 1998.

[13] I. Papautsky, A.B. Frazier, and H. Swerdlow, "A low temperature IC compatible process for fabricating surface micromachined metallic microchannels." MEMS'97.

[14] X.Q. Wang, X.Yang, K. Walsh, and Y.C.Tai, "Gasphase silicon etching with bromine trifluoride", Transducers 97. 